\title{
Monitoring Mobility in Older Adults Using Global Positioning System (GPS) Watches and Accelerometers: A Feasibility Study
}

\author{
Sandra C. Webber and Michelle M. Porter
}

\begin{abstract}
This exploratory study examined the feasibility of using Garmin global positioning system (GPS) watches and ActiGraph accelerometers to monitor walking and other aspects of community mobility in older adults. After accuracy at slow walking speeds was initially determined, 20 older adults $(74.4 \pm 4.2 \mathrm{yr})$ wore the devices for 1 day. Steps, distances, and speeds (on foot and in vehicle) were determined. GPS data acquisition varied from 43 min to over $12 \mathrm{hr}$, with 55\% of participants having more than $8 \mathrm{hr}$ between initial and final data-collection points. When GPS data were acquired without interruptions, detailed mobility information was obtained regarding the timing, distances covered, and speeds reached during trips away from home. Although GPS and accelerometry technology offer promise for monitoring community mobility patterns, new GPS solutions are required that allow for data collection over an extended period of time between indoor and outdoor environments.
\end{abstract}

Keywords: active living, physical activity, aging, walking, driving, community mobility

Maintenance of mobility, defined as "the extent of movement within a person's environment” (Stalvey, Owsley, Sloane, \& Ball, 1999, p. 460), is fundamental to active aging, allowing older adults to continue to lead dynamic and independent lives (World Health Organization, 2007). Mobility can be conceptualized as a continuum from being confined to bed (immobile) to being capable of traveling to distant locations (Patla \& Shumway-Cook, 1999; Stalvey et al.). Research has demonstrated that the ability to move about effectively in one's home and community surroundings is a strong and early predictor of physical disability in older adults (Fried, Bandeen-Roche, Chaves, \& Johnson, 2000; Hirvensalo, Rantanen, $\&$ Heikkinen, 2000). The ability to walk at least short distances reduces the risk of mortality, cardiovascular disease, and mobility limitation (Chang et al., 2004; Newman et al., 2006).

Maintaining or improving walking ability is a consistent aim of rehabilitation for all older adults. In addition to being able to get around the neighborhood on foot, older adults rely heavily on being able to drive or access public transportation

The authors are with the Dept. of Physiology and the Faculty of Kinesiology and Recreation Management, University of Manitoba, Winnipeg, MB, Canada R3T 2N2. 
to continue to be mobile in the community (Dickerson et al., 2007). For those capable of functioning on the higher end of the mobility spectrum, it is impossible in the clinical situation to replicate the environmental demands of specific community settings (Patla \& Shumway-Cook, 1999). Interventions designed to enhance mobility can only be evaluated through assessments that occur in community environments. Questionnaires have been developed to attempt to measure mobility in community-dwelling older adults. Various instruments have been designed to attempt to measure life space, or the spatial extent of travel within one's environment (Baker, Bodner, \& Allman, 2003; May, Nayak, \& Isaacs, 1985; Stalvey et al., 1999; Tinetti \& Ginter, 1990). In addition, the Environmental Analysis of Mobility Questionnaire was developed by Shumway-Cook et al. (2003, 2005) as a self-report mechanism to measure community mobility disability. Both that questionnaire and the life-space tools attempt to consider the many environmental factors (e.g., walking speed and distance requirements, terrain characteristics, weather conditions) and intrapersonal aspects (e.g., functional motor ability, cognitive status) that influence mobility. Although these questionnaires cover many aspects important to mobility, they do not obtain specific information regarding individual trips in the community. For this reason, as well as the fact that questionnaires place a high degree of burden on participants, we were interested in determining whether existing physical activity monitors and global positioning system (GPS) technology might be useful in describing community mobility in older adults.

Accelerometers and pedometers have typically been used to quantify physical activity (see the review by Tudor-Locke \& Myers, 2001). They provide objective measures of steps taken per day and estimates of energy expended during walking. Accelerometer activity counts have been used to categorize physical activity intensity, estimate caloric expenditure, and estimate walking speed (Barnett \& Cerin, 2006; Hendelman, Miller, Baggett, Debold, \& Freedson, 2000; Pober, Staudenmayer, Raphael, \& Freedson, 2006). Although accelerometers have also been used at the wrist and ankle, placement at the hip ensures that they are relatively insensitive to upper body movement and primarily sensitive to capturing information related to walking (Hendelman et al.). Because accelerometers are more sensitive to step detection at slower walking speeds than pedometers, their use has been recommended for quantifying walking in older adults (Culhane, O'Connor, Lyons, \& Lyons, 2005; Le Masurier \& Tudor-Locke, 2003). Accelerometers can provide step counts within user-defined time frames (e.g., every minute) and are capable of storing data for many consecutive days without requiring interaction between the wearer and the device (Trost, McIver, \& Pate, 2005). However, data gathered with an accelerometer are limited to step or activity counts and do not provide information about mobility in different environments. For example, without additional information it is impossible to determine where the steps were taken (e.g., indoors or outdoors, in the home, or in the community).

Satellite-based GPS technology, however, offers potential advantages for studying movement in the community, which may involve outdoor walking, as well as the use of various forms of transportation (Elgethun, Yost, Fitzpatrick, Nyerges, \& Fenske, 2007; Le Faucheur et al., 2007; Phillips, Hall, Esmen, Lynch, \& Johnson, 2001; Rodriguez, Brown, \& Troped, 2005). GPS receivers provide information about location, speed of movement, and elevations encountered and 
may be used to validate physical activity diaries (Rodriguez et al.) and estimate free-living walking capacity (Le Faucheur et al., 2007, 2008). However, GPS reception is frequently interrupted in "urban canyons," under heavy tree canopy, and inside or proximal to large buildings (Elgethun, Fenske, Yost, \& Palcisko, 2003; Phillips et al.; Rodriguez et al.). Receiver interference also occurs in proximity to power substations and microwave ovens (Elgethun et al., 2003). For these reasons another monitor should be used in conjunction with GPS to ensure detection of indoor mobility. Although a few studies have used GPS devices and accelerometers in combination to identify types and locations of outdoor physical activities (Rodriguez et al.; Troped et al., 2008), they have not been used together in older adults. The purpose of this exploratory study was to determine the feasibility of using GPS watches and accelerometers in combination to gather information about walking and other forms of transportation over the course of a day to see whether these technologies may ultimately be useful to measure community mobility patterns in older adults.

\section{Methods}

\section{Participants}

A convenience sample of 20 older adults (68-88 years of age) was recruited to participate in this study. Based on previous studies (Elgethun et al., 2003; Elgethun et al., 2007 ; Phillips et al., 2001; Rodriguez et al., 2005), this sample size was thought to be adequate to provide information regarding a variety of mobility levels and patterns of movement in different locations. All participants lived independently in the community (5 in houses and 15 in apartments), 4 used walking aids, and on average they reported about two current health problems. All participants provided written consent. Ethical approval was granted by the Education/Nursing Research Ethics Board at the University of Manitoba, Winnipeg, MB, Canada.

\section{Equipment}

Garmin Forerunner 205 GPS watches $(53.3 \times 17.8 \times 68.6 \mathrm{~mm}, 77 \mathrm{~g}$; Garmin International Inc., Olathe, KS) were set to "smart recording" mode, resulting in variable recording frequencies and up to $10 \mathrm{hr}$ of data collection. ActiGraph model 7164 dual-mode uniaxial accelerometers $(50.8 \times 38.1 \times 15.2 \mathrm{~mm}, 37.5 \mathrm{~g}$; ActiGraph, LLC, Pensacola, FL) were initialized to record in 1-min epochs and were worn centered over the right thigh in a fitted pouch attached to a belt around the waist. All accelerometers were calibrated by the manufacturer before being used in the study.

\section{Accuracy Tests}

Accuracy of the GPS watches' average speed determination and accelerometer step counts was assessed simultaneously with paced outdoor walking tests conducted by research staff (set distances 200 or $400 \mathrm{~m}$ at $0.45 \mathrm{~m} / \mathrm{s}, 0.67 \mathrm{~m} / \mathrm{s}, 0.89$ $\mathrm{m} / \mathrm{s}, 1.33 \mathrm{~m} / \mathrm{s}, 1.67 \mathrm{~m} / \mathrm{s}$, and $2.08 \mathrm{~m} / \mathrm{s}$ ). The error associated with GPS watches' 
average walking speed and calculated walking speed (known distance/time) was very low (coefficients of variation $[\mathrm{CV}]$ of the measurement errors were $0.8 \%$, $1.5 \%, 2.0 \%, 0.7 \%, 1.4 \%$, and $1.3 \%$ for the respective speeds). These results are consistent with those previously reported for other Garmin units (Barnett \& Cerin, 2006; Le Faucheur et al., 2007). Although ActiGraph accelerometer step counts have been shown to be accurate at speeds as low as $0.9 \mathrm{~m} / \mathrm{s}$ under controlled conditions (Le Masurier, Lee, \& Tudor-Locke, 2004; Le Masurier \& Tudor-Locke, 2003), studies have also found that they significantly underestimate steps at speeds only slightly lower (Esliger et al., 2007; Storti et al., 2008). Our results demonstrated that Actigraph step counts were associated with very low error rates at speeds above $0.90 \mathrm{~m} / \mathrm{s}(\mathrm{CV}=1.1 \%, 0.3 \%$, and $0.8 \%$ for speeds ranging from $0.90-1.33,1.34-1.67$, and $1.68-2.09 \mathrm{~m} / \mathrm{s}$, respectively), but they performed very poorly at lower speeds $(\mathrm{CV}=32.7 \%, 34.7 \%$, and $7.4 \%$ for speeds $\leq 0.45,0.46$ 0.67 , and $0.68-0.89 \mathrm{~m} / \mathrm{s}$ ).

\section{Protocol}

At a mutually convenient time, study staff delivered a Garmin GPS watch (battery fully charged) and an ActiGraph accelerometer to each participant and provided very basic information about how to wear the equipment the following day as the participant went about his or her usual routine. Participants were shown how to position the accelerometer on the belt around their waist, how to power on the watch, and how to start the stopwatch to enable GPS data collection. They were given no further instruction regarding the operation of the equipment and were told to disregard any messages that might appear on the face of the watch during the course of the day. The accelerometers were preprogrammed to start recording at 4:30 a.m. After wearing the devices for 1 day, participants completed a short questionnaire regarding their experiences with the equipment and listed trips taken away from home (by time of day and type of transportation).

\section{Data Processing and Analysis}

GPS data were uploaded to MotionBased software (Sausalito, CA) and saved in formats appropriate for Google Earth (Google, Inc., Mountain View, CA) and SigmaStat (version 3.10, Systat Software Inc., San Jose, CA). Google Earth's mapping service was used to define activity that took place at home or away from home. When time gaps in GPS data collection were identified in the spreadsheet, corresponding locations (pre- and postgap) were determined on the maps.

ActiGraph analysis software was used to determine steps per minute from the cycle counts recorded on the accelerometer. Steps per day were determined in SigmaStat. Accelerometer data were time-matched with GPS data. Outdoor walking distances were identified in the GPS data for time periods that coincided with accelerometer activity. GPS data were also scanned to determine periods of vehicle travel (speed $>3 \mathrm{~m} / \mathrm{s}$ for $>1 \mathrm{~min}$ ) and distances traveled by vehicle. Data were checked for overlap between periods of accelerometer activity and vehicle travel, and steps detected during these periods were subtracted from the daily totals. 


\section{Results}

\section{GPS Data}

Overall, some GPS information was successfully recorded for 18 of the 20 participants (Table 1). The average total duration of GPS data capture was $7.7 \pm 4.1 \mathrm{hr}$, with $55 \%$ of participants successfully acquiring some data for more than $8 \mathrm{hr}$ (range from $43 \mathrm{~min}$ to $12 \mathrm{hr}$ and $19 \mathrm{~min}$ ). Google Earth maps provided detailed information about participants' walking and driving routes. Representative GPS data have been included in Figure 1. The Google Earth map depicts 1 participant driving to a golf course, walking the nine-hole course, and driving away. Speed of movement (in vehicle and on foot) is displayed in the lower part of the figure. Letters $a$ and $c$ correspond to speeds associated with periods of driving, and letter $b$ corresponds to speeds associated with time spent walking on the golf course. Figure 2 depicts a magnified view of movement on the southeast hole on the golf course. The graph displays speed of movement and steps per minute for each minute spent on the hole. Locations coinciding with brief periods of reduced movement speed between the tee box and the green are denoted as $1-4$, which may correspond with separate golf shots and the resultant reduced speed that accompanied preparing to hit the golf ball. The steps accumulated over each

Table 1 Details of GPS Data Capture for 20 Older Adults Over 1 Day

\begin{tabular}{lcc}
\hline Participant & $\begin{array}{c}\text { Delay in GPS capture after } \\
\text { watch turned on (min) }\end{array}$ & $\begin{array}{c}\text { Duration between initial and final } \\
\text { GPS data acquisition (hr) }\end{array}$ \\
\hline 1 & 79.88 & 10.07 \\
2 & 319.68 & 1.12 \\
3 & 9.93 & 2.02 \\
4 & 5.42 & 11.65 \\
5 & No GPS data & No GPS data \\
6 & 67.33 & 10.55 \\
7 & 3.17 & 12.32 \\
8 & 11.87 & 11.50 \\
9 & 0 & 0.72 \\
10 & 307.88 & 2.88 \\
11 & 54.67 & 3.38 \\
12 & 18.98 & 11.65 \\
13 & 12.98 & 7.63 \\
14 & No GPS data & No GPS data \\
15 & Unknown & 9.93 \\
16 & 18.10 & 4.46 \\
17 & 37.28 & 8.58 \\
18 & 182.18 & 8.22 \\
19 & 64.46 & 10.53 \\
20 & 67.00 & 10.67 \\
\hline
\end{tabular}



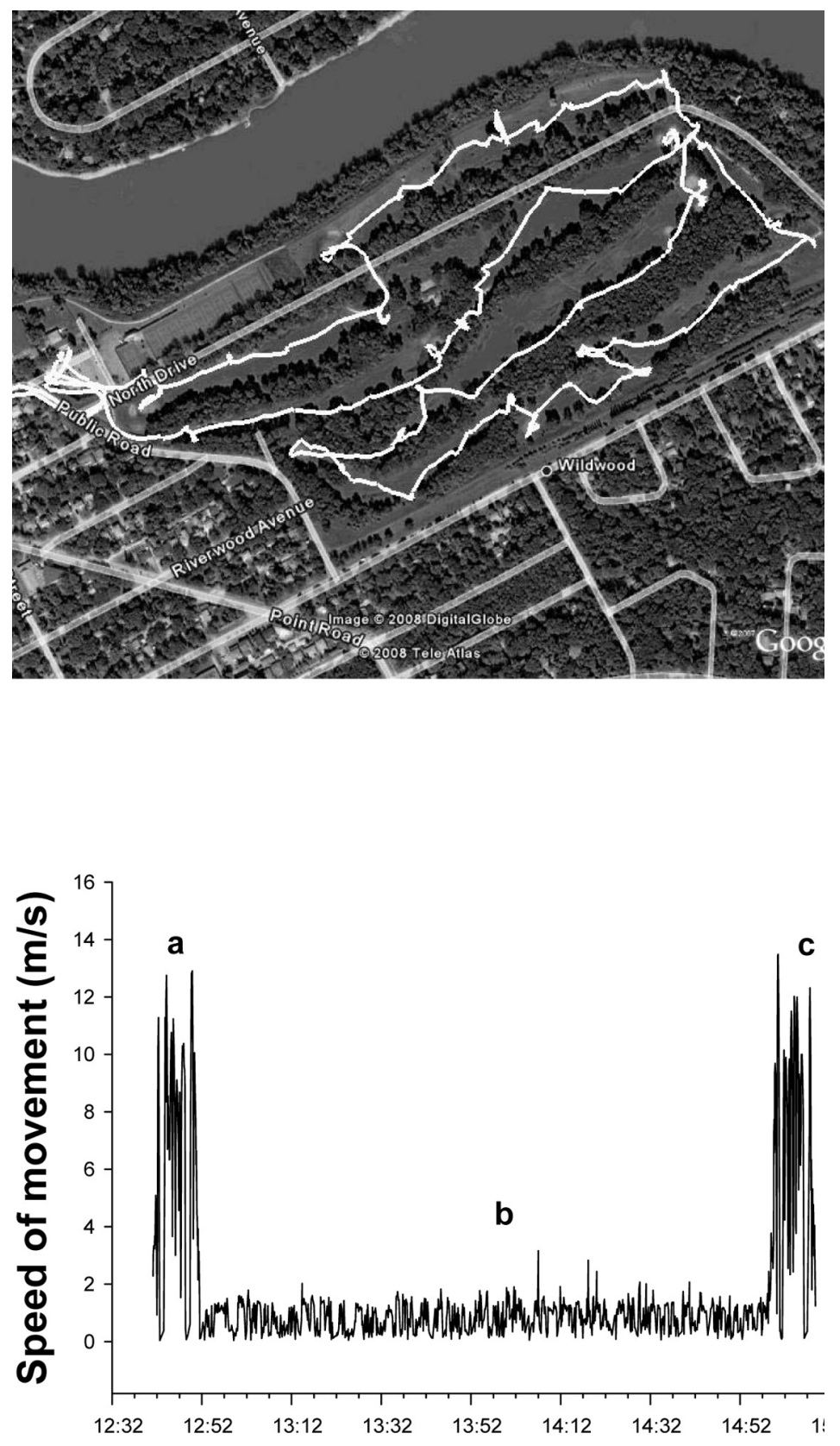

Figure 1 - Google Earth map (top) and speed data (bottom) obtained from part of the GPS data for 1 participant. Letters $a$ and $c$ correspond to driving to and from the golf course, and letter $b$, to walking the nine-hole course. Satellite image courtesy of DigitalGlobe. 

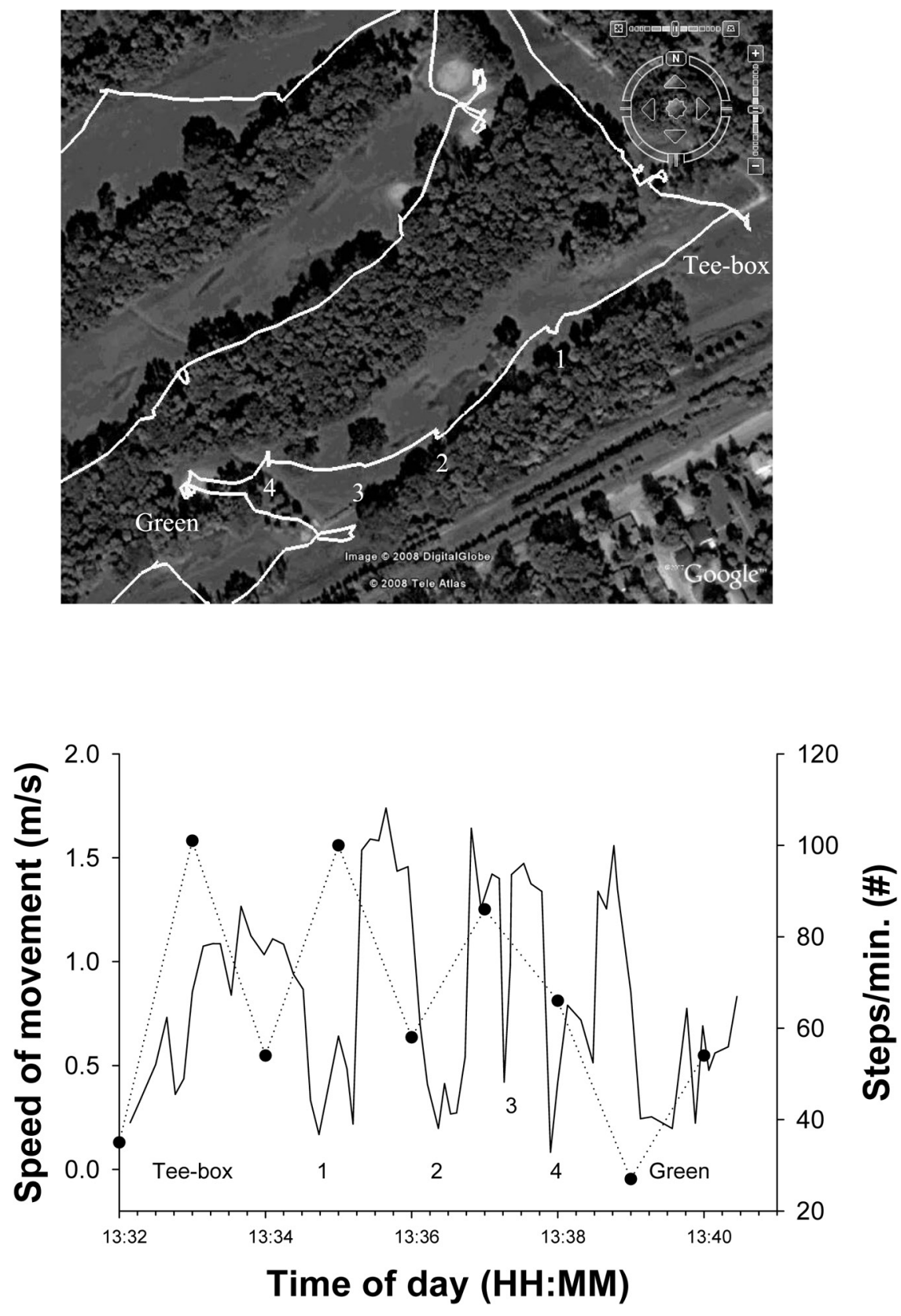

Figure 2 - Google Earth map (top) and speed/steps data (bottom) obtained from the southeast hole on the golf course. Numbers 1-4 represent brief periods of reduced speed that may coincide with individual shots on the hole. Steps per minute are plotted for each minute (connected by the dotted line). Satellite image courtesy of DigitalGlobe. 
minute are plotted at the end of the minute (e.g., step number plotted at 13:36 represents the sum of steps taken between 13:35 and 13:36).

Although it was possible for the GPS unit to acquire a signal when some participants were inside some buildings (which presumably depended on the participant's location, type of building construction, and the number of windows), the signal was frequently lost when participants went indoors. Delays in GPS data acquisition were encountered at start-up (turning the GPS watch on indoors resulted in "cold," indoor starts) and when participants left buildings.

Data from the 8 older adults who acquired more than $10 \mathrm{hr}$ of GPS data (GPS watch start time to stop time) were analyzed to investigate the variable datarecording rate that resulted from using the "smart recording" setting on the Garmin watch. For these 8 participants, 97\% of successive data points were recorded within $1 \mathrm{~min}$ of each other. Analysis of these data points demonstrated that the average duration between GPS data points was $10.35 \pm 1.58 \mathrm{~s}$. Median times between data points varied between 6 and $8 \mathrm{~s}$, a frequency similar to that used in other GPS data-acquisition studies (Phillips et al., 2001; Rodriguez et al., 2005).

\section{Accelerometer Data}

Accelerometer data indicated that participants' step counts ranged from 3,564 to 23,888 steps per day $(10,012 \pm 5,026)$, with the longest walk for each participant (occurring indoors or outdoors) lasting from 8 to $53 \mathrm{~min}(26.2 \pm 13.9 \mathrm{~min}$ ).

\section{Time-Matched GPS and Accelerometer Data}

Average walking speed was determined for participants who walked for more than 10 min consecutively outdoors when accelerometer and GPS data were successfully captured simultaneously. Average walking speed for the 11 participants who met these criteria was $1.27 \pm 0.32 \mathrm{~m} / \mathrm{s}$ (range $0.76-1.72 \mathrm{~m} / \mathrm{s}$ ). Analysis of the periods of vehicle travel (determined by velocity threshold) indicated that accelerometers recorded $793 \pm 1,205$ steps erroneously while participants traveled in vehicles.

\section{Questionnaires}

All participants rated the GPS watch and accelerometer easy to use and reported that they wore the equipment for the full day. In addition, all reported that the accelerometer was comfortable to wear on a belt. Nineteen of 20 rated the GPS watch comfortable to wear. On the questionnaires, no participants reported any concerns with using the equipment or with having their mobility tracked. Although there are many issues emerging relating to the ethics of using GPS to track human movement, concerns largely focus on situations in which the monitor is in a position of power over the person being monitored (e.g., parents monitoring children, law-enforcement officials monitoring criminals, and caregivers monitoring people with dementia; Michael, McNamee, \& Michael, 2006). In describing the methods of data collection to our participants, we made it clear that we would potentially be able to monitor their location and speed throughout the entire day. 


\section{Discussion}

GPS receivers, which are capable of providing information about how often people leave their homes, when they leave their homes, where they travel, how they travel (on foot or in vehicle), and how quickly they move, offer promise for ultimately being able to objectively monitor community mobility in older adults. They collect detailed information about individual trips away from home that cannot be obtained with existing questionnaires. However, results from this study indicate that advances in technology are required before it will be possible to gather complete data over the course of many days, which may then be used to describe older adults' mobility patterns.

Previous studies have demonstrated that GPS devices function well when used during discrete (20- to 45-min) outdoor bouts such as walking (Le Faucheur et al., 2007, 2008; Troped et al., 2008), cycling, in-line skating, or in a vehicle (Troped et al.). For example, GPS receivers have successfully been used to measure outdoor walking capacity (in terms of distances walked and duration of walk and rest periods) in older adults with peripheral arterial disease (Le Faucheur et al., 2008). In addition, it would be anticipated that GPS devices would function well and return detailed information about extended bouts of outdoor activity (e.g., golfing, orienteering).

However, difficulties have been encountered in GPS studies that attempted to monitor people's movements and locations over much longer periods of time and between indoor and outdoor positions. Rodriguez et al. (2005) attempted to use spatial data from GPS units to complement physical activity measures detected by accelerometers. Bouts of physical activity that were easily identified in the accelerometer data were time-matched to the GPS data to determine and classify the location of physical activity bouts (e.g., indoors, outdoors, and location relative to street and land use). In that 3-day study of 35 young adults, 11 participants had incomplete GPS data. In another study, conducted by Phillips et al. (2001), GPS units were used to track 25 participants' locations to assess potential environmental exposure over a 24-hr period (Phillips et al.). Data were captured for only $30 \%$ of the total monitoring time, and 14 participants had less than $8 \mathrm{hr}$ of data. Many of their waist-worn GPS units suffered apparent battery failure. In our study, with a newer device, GPS information was collected for over $8 \mathrm{hr}$ in $55 \%$ of participants. However, within this time frame a number of participants experienced gaps in GPS signal acquisition that may represent a lost signal related to prolonged indoor time or could represent failure of the GPS unit to collect information related to outdoor movement. It is known that some participants experienced significant delays in satellite lock-in on initially starting the watches if they presumably remained indoors for a long period of time after waking in the morning (see Table 1, Participants 2,10, and 18), and this may also have occurred midday after they exited buildings. Other problems including premature battery failure and accidental bumping of the buttons on the face of the watch may also have contributed to lost data.

Despite the problems with GPS data acquisition, GPS technology provides the opportunity to collect much more detailed and relevant information about mobility in community environments (e.g., where an individual walks, how quickly he or she must cross a street, what degree of incline is encountered) than 
step or activity counts monitored by accelerometers or pedometers. As is evident in Figure 2, GPS information gathered as an individual walks provides much more detailed information about the experience than step counts measured by an accelerometer. When using this equipment with older adults, it is also important to consider the fact that accelerometer accuracy may be compromised by age-related changes in mechanical gait parameters (e.g., slower, shuffling gait or changes related to using a walking aid) that influence acceleration in the vertical plane. GPS measurements of speed and displacement would not be sensitive to these changes.

Using GPS devices and accelerometers together provides the most complete information about mobility in community environments. Because there is a potential for incomplete GPS data acquisition, especially when mobility is monitored over longer periods of time, using accelerometers in combination with GPS devices provides the assurance that at least some form of mobility information will be acquired even when the satellite signal is not available. For example, because GPS units do not function well indoors, the addition of accelerometers provides the opportunity to acquire information about the number and timing of steps taken in the home and other buildings. From this, it is at least possible to know when an individual was mobile or stationary during their time indoors. This same functionality is also provided if the individual is outdoors and the GPS signal is lost (e.g., in an urban canyon).

Although accelerometers are more sensitive than mechanical pedometers (Le Masurier et al., 2004; Le Masurier \& Tudor-Locke, 2003) and their use has been recommended in the older population (Marsh, Vance, Frederick, Hesselmann, \& Rejeski, 2007), the greater sensitivity of the device can result in false step recordings during travel in a vehicle. When GPS receivers are used in conjunction with accelerometers, periods of vehicle travel can be identified and erroneous step or activity counts can be removed from the data. In addition, using a GPS device in combination with an accelerometer allows for detection of outdoor gait velocities below the threshold for accelerometers $(0.86 \mathrm{~m} / \mathrm{s})$, which occur frequently in frail older people (Lindemann et al., 2007).

There are many different handheld and watch- or phone-based GPS receivers available on the market today. Performance characteristics vary depending on sampling frequency, lock-in time to acquire or reacquire the GPS signal, battery life, size, weight, method of wearing the device, and cost. Because most of these devices were not developed for research purposes, no systematic comparison of performance features among GPS units has been done. Many of the published studies to date that have used GPS to track human movement required participants to wear relatively large, cumbersome devices. GPS data recorders have been attached on the belt along with external battery packs and antennae fixed on the shoulder (Phillips et al., 2001). Alternatively, GPS data recorders, batteries, and antennae have been sewn into vests and bib overalls for children to wear (Elgethun et al., 2003). Other studies have positioned the GPS devices in a small backpack with the antenna attached to a shoulder strap (Le Faucheur et al., 2008; Troped et al., 2008). We chose GPS watches for use in this study because of their relatively small size, light weight, and unobtrusive nature. Garmin GPS watches were designed to be used by athletes to track distances and speeds during outdoor workouts. Despite the 10-hr battery life, they are not intended to be used for 
extended periods of time with wearers moving between indoor and outdoor locations without stopping to allow the watch to lock in to satellites each time they go outdoors. To minimize influences on normal everyday mobility, we decided that participants should not be instructed to allow time for satellite lock-in on going outdoors during the day. This procedure likely resulted in lost data in some instances and explains the relatively frequent occurrence of gaps in GPS data collection. In addition, battery life could have been maximized by discontinuing GPS data collection when indoors and restarting on going outdoors. However, we felt that this would have added unreasonable burden to the participants, and they might not have remembered to restart the watch when returning outdoors, which also would have resulted in missing data.

In future studies participants could be given more detailed instructions about using the GPS watch to obtain more complete GPS data. For example, participants could be told to stand stationary for $2-3$ min to ensure satellite lock-in every time they exit a building or emerge from an area consisting of tall buildings or substantial tree cover. GPS devices could be turned off when participants go indoors to try to prolong battery life and maximize data collection. In this case, of course, it would be essential to use another mobility-monitoring device such as an accelerometer to be able to gain some information about relative movement inside buildings. However, researchers must be aware that placing these requirements on individuals may in fact influence participants' mobility patterns (in terms of the number and duration of trips taken from home) or result in missing data (e.g., if people do not remember to turn the devices back on after they have been turned off).

In conclusion, although combined GPS and accelerometer technologies have been used successfully to gather detailed information about discrete bouts of outdoor activity (physical endeavors, as well as driving), the same success has not been realized in studies that have attempted to monitor functional everyday human movement over an extended period of time. Although these technologies offer the potential to accurately monitor community mobility patterns in older adults, some issues still need to be addressed before this is realized.

\section{Acknowledgments}

Support was provided by the Canadian Institutes of Health Research (Institute of Aging Mobility in Aging Grant and a fellowship). We acknowledge Heather Ash and Satoru Nakagawa for technical assistance and Dr. Verena Menec for the accelerometers.

\section{References}

Baker, P.S., Bodner, E.V., \& Allman, R.M. (2003). Measuring life-space mobility in community-dwelling older adults. Journal of the American Geriatrics Society, 51(11), $1610-1614$.

Barnett, A., \& Cerin, E. (2006). Individual calibration for estimating free-living walking speed using the MTI monitor. Medicine and Science in Sports and Exercise, 38(4), 761-767.

Chang, M., Cohen-Mansfield, J., Ferrucci, L., Leveille, S., Volpato, S., de Rekeneire, N., et al. (2004). Incidence of loss of ability to walk 400 meters in a functionally limited older population. Journal of the American Geriatrics Society, 52(12), 2094-2098. 
Culhane, K.M., O'Connor, M., Lyons, D., \& Lyons, G.M. (2005). Accelerometers in rehabilitation medicine for older adults. Age and Ageing, 34(6), 556-560.

Dickerson, A.E., Molnar, L.J., Eby, D.W., Adler, G., Bedard, M., Berg-Weger, M., et al. (2007). Transportation and aging: A research agenda for advancing safe mobility. The Gerontologist, 47(5), 578-590.

Elgethun, K., Fenske, R.A., Yost, M.G., \& Palcisko, G.J. (2003). Time-location analysis for exposure assessment studies of children using a novel global positioning system instrument. Environmental Health Perspectives, 111(1), 115-122.

Elgethun, K., Yost, M.G., Fitzpatrick, C.T., Nyerges, T.L., \& Fenske, R.A. (2007). Comparison of global positioning system (GPS) tracking and parent-report diaries to characterize children's time-location patterns. Journal of Exposure Science \& Environmental Epidemiology, 17(2), 196-206.

Esliger, D.W., Probert, A., Gorber, S.C., Bryan, S., Laviolette, M., \& Tremblay, M.S. (2007). Validity of the Actical accelerometer step-count function. Medicine and Science in Sports and Exercise, 39(7), 1200-1204.

Fried, L.P., Bandeen-Roche, K., Chaves, P.H., \& Johnson, B.A. (2000). Preclinical mobility disability predicts incident mobility disability in older women. The Journals of Gerontology. Series A, Biological Sciences and Medical Sciences, 55(1), M43-M52.

Hendelman, D., Miller, K., Baggett, C., Debold, E., \& Freedson, P. (2000). Validity of accelerometry for the assessment of moderate intensity physical activity in the field. Medicine and Science in Sports and Exercise, 32(9, Suppl) S442-S449.

Hirvensalo, M., Rantanen, T., \& Heikkinen, E. (2000). Mobility difficulties and physical activity as predictors of mortality and loss of independence in the community-living older population. Journal of the American Geriatrics Society, 48(5), 493-498.

Le Faucheur, A., Abraham, P., Jaquinandi, V., Bouye, P., Saumet, J.L., \& Noury-Desvaux, B. (2007). Study of human outdoor walking with a low-cost GPS and simple spreadsheet analysis. Medicine and Science in Sports and Exercise, 39(9), 1570-1578.

Le Faucheur, A., Abraham, P., Jaquinandi, V., Bouye, P., Saumet, J.L., \& Noury-Desvaux, B. (2008). Measurement of walking distance and speed in patients with peripheral arterial disease: A novel method using a global positioning system. Circulation, 117(7), 897-904.

Le Masurier, G.C., Lee, S.M., \& Tudor-Locke, C. (2004). Motion sensor accuracy under controlled and free-living conditions. Medicine and Science in Sports and Exercise, 36(5), 905-910.

Le Masurier, G.C., \& Tudor-Locke, C. (2003). Comparison of pedometer and accelerometer accuracy under controlled conditions. Medicine and Science in Sports and Exercise, 35(5), 867-871.

Lindemann, U., Najafi, B., Zijlstra, W., Hauer, K., Muche, R., Becker, C., et al. (2008). Distance to achieve steady state walking speed in frail elderly persons. Gait \& Posture, 27(1), 91-96.

Marsh, A.P., Vance, R.M., Frederick, T.L., Hesselmann, S.A., \& Rejeski, W.J. (2007). Objective assessment of activity in older adults at risk for mobility disability. Medicine and Science in Sports and Exercise, 39(6), 1020-1026.

May, D., Nayak, U.S., \& Isaacs, B. (1985). The life-space diary: A measure of mobility in old people at home. International Rehabilitation Medicine, 7(4), 182-186.

Michael, K., McNamee, A., \& Michael, M.G. (2006). The emerging ethics of humancentric GPS tracking and monitoring. In Proceedings of the International Conference on Mobile Business, Denmark, 34. Retrieved September 16, 2008, from the University of Wollongong, Faculty of Informatics, Web site: http://ro.uow.edu.au/infopapers/385

Newman, A.B., Simonsick, E.M., Naydeck, B.L., Boudreau, R.M., Kritchevsky, S.B., Nevitt, M.C., et al. (2006). Association of long-distance corridor walk performance with mortality, cardiovascular disease, mobility limitation, and disability. Journal of the American Medical Association, 295(17), 2018-2026. 
Patla, A.E., \& Shumway-Cook, A. (1999). Dimensions of mobility: Defining the complexity and difficulty associated with community mobility. Journal of Aging and Physical Activity, 7(1), 7-19.

Phillips, M.L., Hall, T.A., Esmen, N.A., Lynch, R., \& Johnson, D.L. (2001). Use of global positioning system technology to track subject's location during environmental exposure sampling. Journal of Exposure Analysis and Environmental Epidemiology, 11(3), 207-215.

Pober, D.M., Staudenmayer, J., Raphael, C., \& Freedson, P.S. (2006). Development of novel techniques to classify physical activity mode using accelerometers. Medicine and Science in Sports and Exercise, 38(9), 1626-1634.

Rodriguez, D.A., Brown, A.L., \& Troped, P.J. (2005). Portable global positioning units to complement accelerometry-based physical activity monitors. Medicine and Science in Sports and Exercise, 37(11, Suppl) S572-S581.

Shumway-Cook, A., Patla, A., Stewart, A., Ferrucci, L., Ciol, M.A., \& Guralnik, J.M. (2003). Environmental components of mobility disability in community-living older persons. Journal of the American Geriatrics Society, 51(3), 393-398.

Shumway-Cook, A., Patla, A., Stewart, A.L., Ferrucci, L., Ciol, M.A., \& Guralnik, J.M. (2005). Assessing environmentally determined mobility disability: Self-report versus observed community mobility. Journal of the American Geriatrics Society, 53(4), 700-704.

Stalvey, B.T., Owsley, C., Sloane, M.E., \& Ball, K. (1999). The Life Space Questionnaire: A measure of the extent of mobility of older adults. Journal of Applied Gerontology, 18(4), 460-478.

Storti, K.L., Pettee, K.K., Brach, J.S., Talkowski, J.B., Richardson, C.R., \& Kriska, A.M. (2008). Gait speed and step-count monitor accuracy in community-dwelling older adults. Medicine and Science in Sports and Exercise, 40(1), 59-64.

Tinetti, M.E., \& Ginter, S.F. (1990). The nursing home life-space diameter. A measure of extent and frequency of mobility among nursing home residents. Journal of the American Geriatrics Society, 38(12), 1311-1315.

Troped, P.J., Oliveira, M.S., Matthews, C.E., Cromley, E.K., Melly, S.J., \& Craig, B.A. (2008). Prediction of activity mode with global positioning system and accelerometer data. Medicine and Science in Sports and Exercise, 40(5), 972-978.

Trost, S.G., McIver, K.L., \& Pate, R.R. (2005). Conducting accelerometer-based activity assessments in field-based research. Medicine and Science in Sports and Exercise, 37(11, Suppl) S531-S543.

Tudor-Locke, C.E., \& Myers, A.M. (2001). Challenges and opportunities for measuring physical activity in sedentary adults. Sports Medicine (Auckland, N.Z.), 31(2), 91100.

World Health Organization. (2007). Global age-friendly cities: A guide. Geneva, Switzerland: WHO Press. 